\title{
The validity of dietary assessment methods to accurately measure energy intake in children and adolescents who are overweight or obese: a systematic review
}

\author{
Jacqueline L. Walker $\mathbb{D}^{1} \cdot$ Stephen Ardouin ${ }^{1} \cdot$ Tracy Burrows $\mathbb{D}^{2}$ \\ Received: 28 June 2017 / Revised: 10 September 2017 / Accepted: 25 September 2017 / Published online: 20 December 2017 \\ (c) The Author(s) 2018. This article is published with open access
}

\begin{abstract}
The accurate assessment of energy intake in children and adolescents is an important outcome measure for clinical and population-based research. This systematic review aimed to determine the validity of dietary assessment methods to measure energy intake in children and adolescents who are classified as overweight or obese by comparison with doubly labelled water. Five electronic databases were searched using keywords. Of the 5263 papers identified, seven papers describing six studies met the inclusion criteria. Studies were included in the review if participants were classified as overweight or obese, aged 0-18 years old, if they estimated energy intake via a dietary assessment method and if they compared this to total energy expenditure measured via the doubly labelled water method. All studies were cross-sectional in nature, and each used one dietary assessment method, including 14-day-food record (FR; $n=1), 24 \mathrm{~h}$ dietary recall $(n=1)$, 8-day FR $(n=1)$, 9day FR $(n=1), 3$-day FR $(n=1)$ and diet history interview $(n=1)$. Sample sizes ranged from 9 to 59 participants, with the majority of studies including less than 30 participants $(n=4)$. Mis-reporting was evident in all of the studies, with underreporting $(n=5)$ more frequent than over-reporting $(n=1)$. Findings from this review suggest that a 24 -h dietary recall and diet history interview were the most accurate methods at the group level for children aged 4-14 years, where the parent or combined child and parent were the reporters.
\end{abstract}

\section{Introduction}

Overweight and obesity in childhood and adolescence is a critically important health care issue, with over 42 million overweight children globally under the age of 5 [1], and more than 1 million Australian children and adolescents (27.4\%) classified as overweight or obese ${ }^{2}$. It is well documented that dietary energy intake (EI) is one of the major contributors to positive energy balance in children and adolescents, in addition to physical activity behaviours, biological factors and environmental influences [3]. Accurate measurement of EI in all children and adolescents is an important outcome when

Jacqueline L. Walker

j.walker3@uq.edu.au

1 School of Human Movement and Nutrition Sciences, The University of Queensland, St Lucia, QLD, Australia

2 Priority Research Centre for Physical Activity and Nutrition and the School of Health Sciences, Faculty of Health and Medicine, University of Newcastle, Newcastle, NSW, Australia monitoring population trends, interpreting clinical outcomes, and refining energy-based recommendations. Yet, dietary assessment of EI in children and adolescents who are overweight or obese remains problematic, and the most appropriate method or tool to use continues to be unclear. Children and adolescents who are overweight or obese have repeatedly reported to consume similar [4], and even fewer calories than their normal weight counterparts [5] and it is uncertain whether this is due to error in the type of methodology used or other factors such as socially desirable reporting, day-to day variation in EI or difficulty estimating portion sizes [6]. Despite the challenges associated with dietary assessment methodology and the complexity of capturing dietary intake in children, the determination of self- or proxy-reported intake remains an important element of health research [7].

A variety of dietary assessment methods are available for assessing energy adequacy and intake in children and adolescents [8]. The advantages, disadvantages and appropriate applications of these dietary assessment methods are extensively explained elsewhere [9]. It is essential, however, that valid and reliable tools are available for use in various populations, including children and adolescents 
who are overweight or obese. Validity describes the degree to which a dietary assessment method measures what it is intended to measure [10]. As measuring absolute validity poses significant practical difficulties, research has often focused on relative validity [11]. Relative validity can be described as the direct comparison of reported intake from a dietary assessment method against an alternative validation method (often known as the reference method), which has a greater degree of demonstrated validity [11]. Doubly labelled water (DLW) is considered a gold-standard method used to calculate total energy expenditure (TEE) in freeliving humans [12]. It has been validated for use in populations ranging from infants to the elderly [12], and has been shown to be accurate to within 1-2\% [13]. The comparison of estimated EI to TEE (measured via the DLW method) can be made because it is fair to assume that TEE equals EI in individuals who are in a state of energy balance, and that the energy used for growth in children is negligible at $2 \%$ of EI [14], hence does not need to be accounted for in energy balance studies [10].

A comprehensive systematic review conducted in 2010 by Burrows et al. [15] investigated the validity of dietary assessment methods to accurately estimate EI in children when compared to DLW. The previous paediatric review identified only 2 out of the 15 included studies that included a population group who were overweight or obese [15]. Results demonstrated that EI was under-reported in overweight and obese children, and that these children were more likely to under-report when compared to their nonobese peers [15]. Conclusions focused on recommendations for the paediatric population according to age range and the reporter of intake [15]; however, specific recommendations according to weight status were not covered.

The underlying goal when assessing dietary intake in research or clinical settings is to be able to determine validity and accuracy of the outcome of interest, using a practical, appropriate and low participant burden dietary assessment method [16]. What remains unclear is what dietary assessment methods are valid to use for estimating EI in children and adolescents who are overweight or obese, given the known differences in reporting [15]. The aim of this review was to gather evidence to determine the validity of dietary assessment methods to estimate EI in overweight and obese children and adolescents.

\section{Materials and methods}

The electronic databases PubMed, Scopus, Science Direct, CINAHL and the Cochrane Library were searched for relevant literature using keywords and combinations of them: child; adolescent; overweight; obesity; paediatric obesity; EI; dietary assessment; nutritional assessment; food frequency questionnaire; dietary recall; food diary; food record; and DLW. Articles were retrieved from the beginning of database archives until November 2016. The Preferred Reporting Items for Systematic Reviews and MetaAnalyses (PRISMA) Statement was used as a guide in this review [17]. Articles were assessed for inclusion in three stages. The first stage involved the screening of titles and abstracts by two independent reviewers (S.A. and J.L.W.). In the second stage, full text copies of the articles were reviewed and evaluated for inclusion, again by two independent reviewers (S.A. and J.L.W.). Articles were included if they met all of the following criteria: (1) included participants were children or adolescents ( $0-18$ years) who were classified as overweight or obese (using any relevant classification method); (2) estimated EI by use of a dietary assessment method; (3) used DLW to estimate and report TEE; and (4) made comparisons between EI and TEE, or comparisons were able to be made from reported data. If articles included participants in the specified age range of both healthy/normal weight and overweight or obese, and the results between the different weight groups could be distinguished, the article was deemed eligible for inclusion. Articles were excluded if: (1) the children or adolescents who were overweight or obese had any medical condition that was non-obesity-related; and (2) they were not published in English. The reference lists of articles identified in the second stage of screening were also hand-searched for any further relevant articles. After stage two, all articles identified for potential inclusion were assessed according to the National Health and Medical Research Council (NHMRC) levels of evidence [18], which is an Australian resource, and reflects the risk of bias in the presented results that is related to the study design. The evidence within the included articles was then assessed for quality using the Academy of Nutrition and Dietetics Evidence Analysis Manual, Quality Criteria Checklist for Primary Research [19]. This tool assesses both the relevance and validity of evidence across 11 domains and identifies potential bias by examining inclusion/exclusion criteria, participant selection, generalisability, data collection, data analysis and presentation of outcomes. Studies are given a positive, negative or neutral rating based on the final scores across the 11 domains. No studies were excluded based on quality in stage two.

During critical analysis of included articles, data were extracted and compared to determine the extent of valid reporting. Data included values for estimated EI, measured TEE, reporting status, limits of agreement (LOA) and methodical details and processes (see Tables 1-3). Definitions from previous literature regarding reporting status were used in this review, which compare the level of accuracy of reported EI to measured TEE (EI/TEE), taking into account the $95 \%$ confidence limits of the expected ratio 


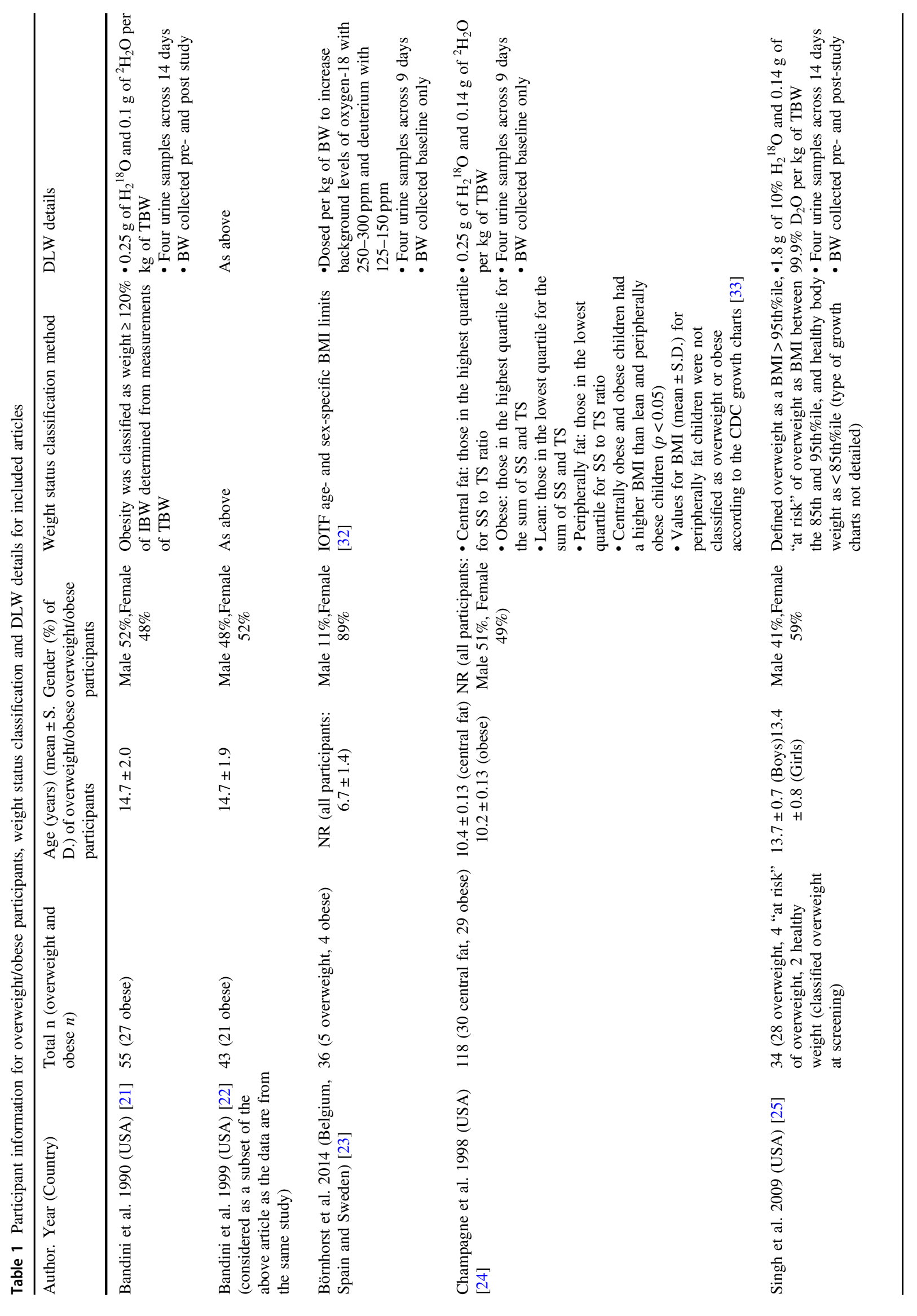


of 1.00 [20]. Adequate reporters are defined as those where the ratio of EI to TEE was within the $95 \%$ confidence limits of 0.84-1.16; over-reporters are those $>1.16$; and underreporters are those $<0.84$ [20]. Figure 1 gives an overview of the searching and inclusion processes.

\section{Results}

A total of 5684 articles were identified from the initial search of databases, as identified in Fig. 1. Seven articles reporting on six studies were included in the final analyses. Bandini et al. reported study outcomes in an article published in 1990 [21] and again for a subset of participants in a second article published in 1999 [22]. Due to the significant homogeneity in the data sets, results from the article published in 1999 [22] are presented and considered as a subset of the article published in 1990 [21], so as to not count results twice. Across the six included studies, the date of publication ranged from 1990 to 2014, and a total of 174 subjects met the weight classification inclusion criteria, ranging in age from 3 to 18 years [21-27]. Sample sizes of children and adolescents who were overweight or obese ranged from 9 to 59 participants, with the majority of studies including less than 30 participants $(n=4)$ [21,23,26,27]. All studies were cross-sectional in design, and were level III-2 evidence, given there were non-blinded comparisons with a reference standard (DLW) in selected populations (i.e., the included participants were unlikely to have been a representative sample of all children who are overweight or obese) [18]. Quality assessment: all studies had a positive study quality rating, meaning studies were well-executed, had sound scientific investigations, were applicable to practice and the conclusions are likely valid, despite known limitations (both practical and financial) regarding blinding of participants, data collectors and researchers [19]. Two studies focused on adolescents (12-18 years) [21,25], three on children (8.3-11 years) $[23,24,27]$ and one on young children ( $3-5$ years) [26]. The way in which weight status (overweight or obese) was classified varied across studies, and included the use of body mass index (BMI) $z$-score or percentile data (countryspecific or international) $[23,25,27]$, weight for height $z$ score data (country-specific) [26], triceps and subscapular skinfold measurements [24] and calculations of ideal body weight from total body water measurements [21]. Three studies included a comparator group (children who were within a healthy weight range) $[21,23,24]$; however, only one of these studies actually made comparisons between this group's reporting status and the children or adolescents who were overweight or obese [21]. The DLW collection period ranged from 7 to 14 days, and two studies did not measure body weight both pre and post the DLW study 


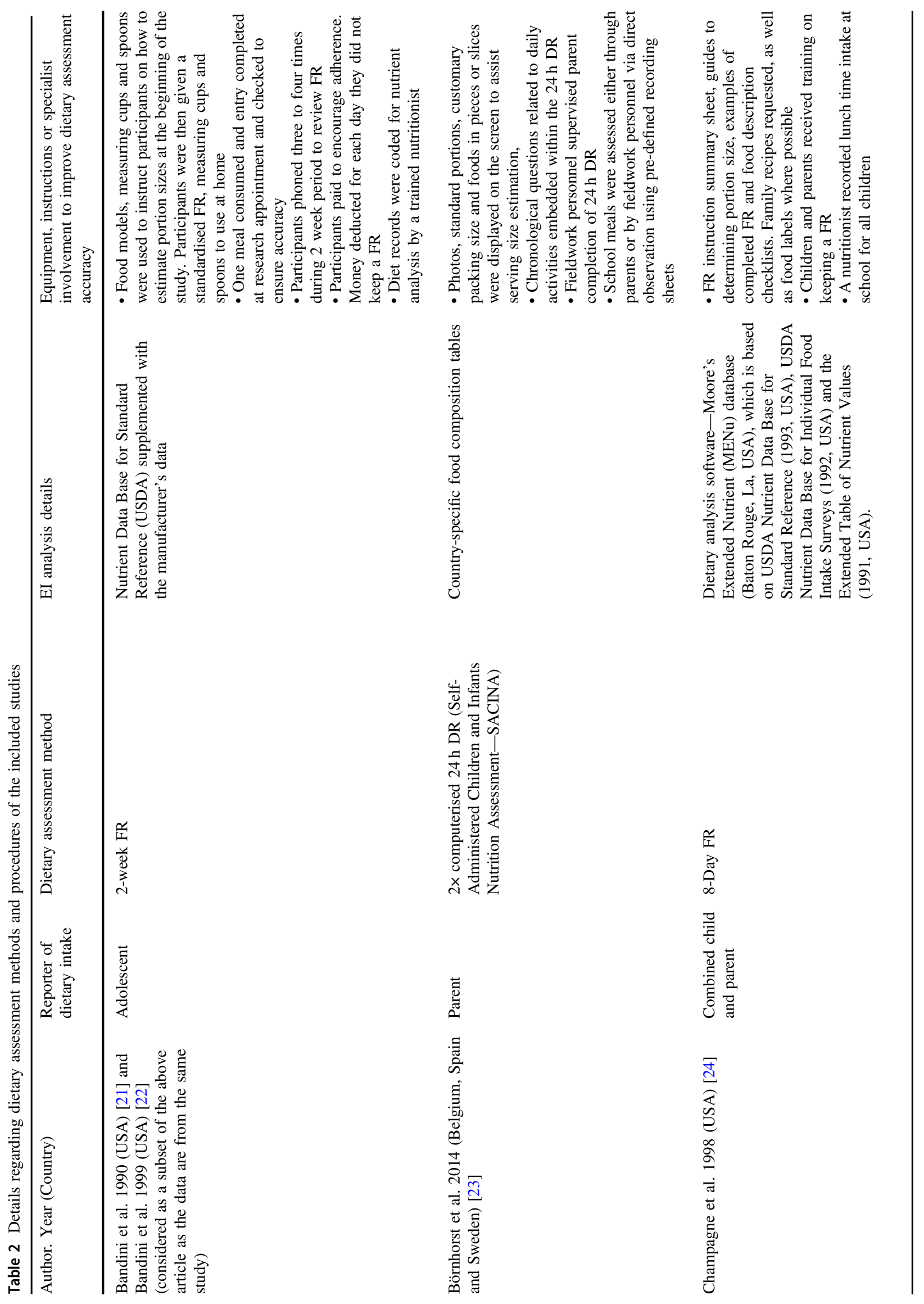




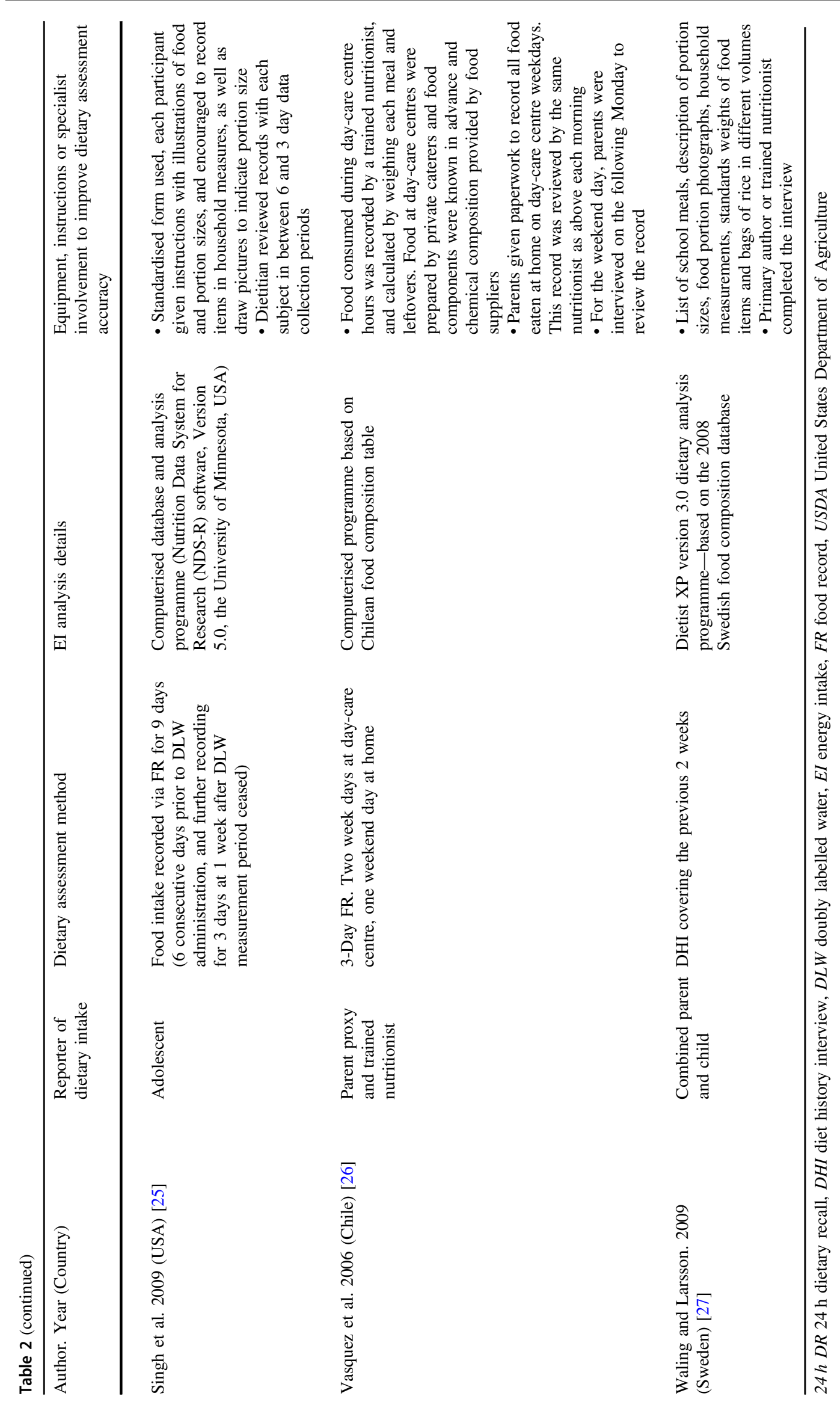




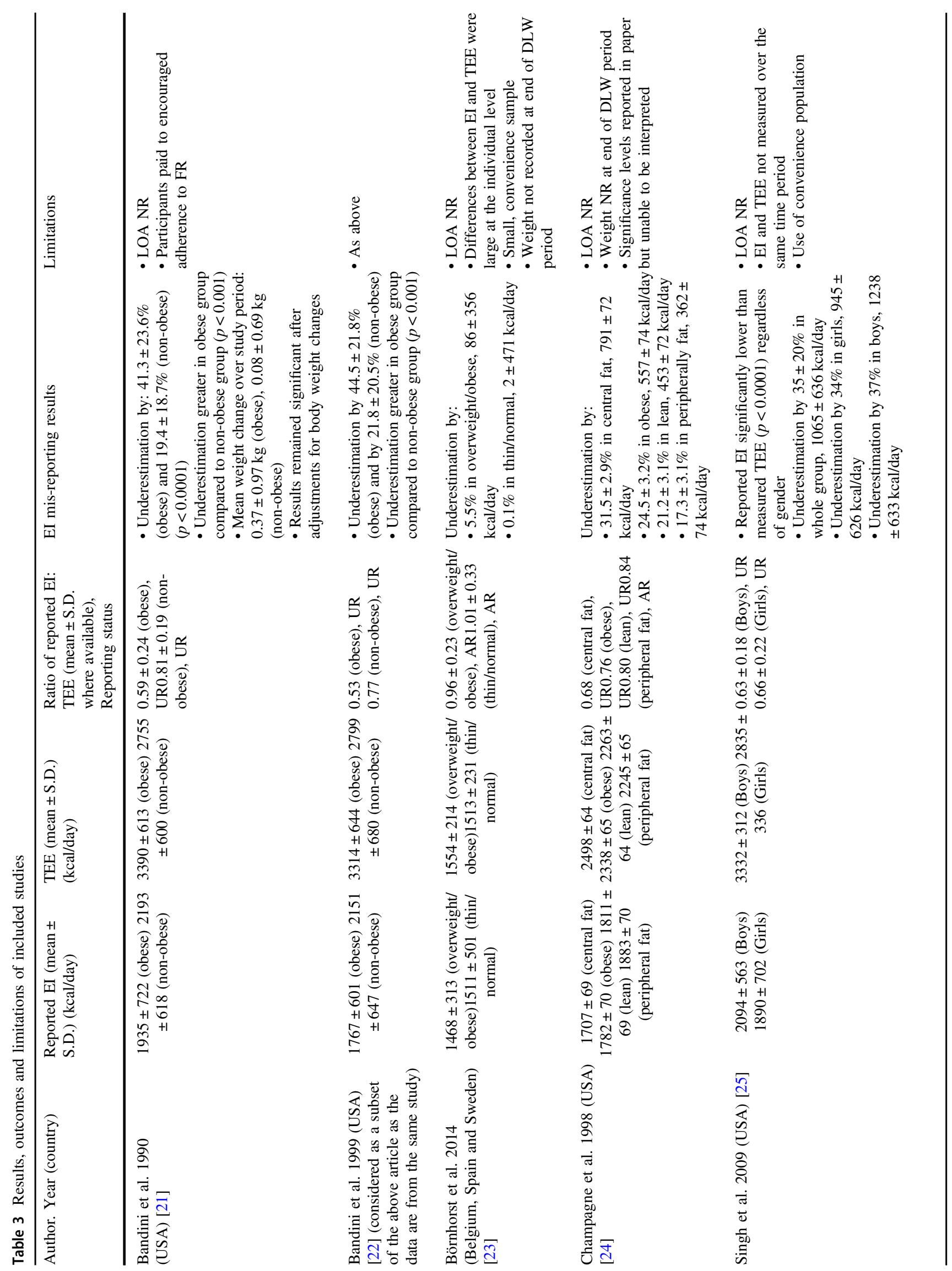




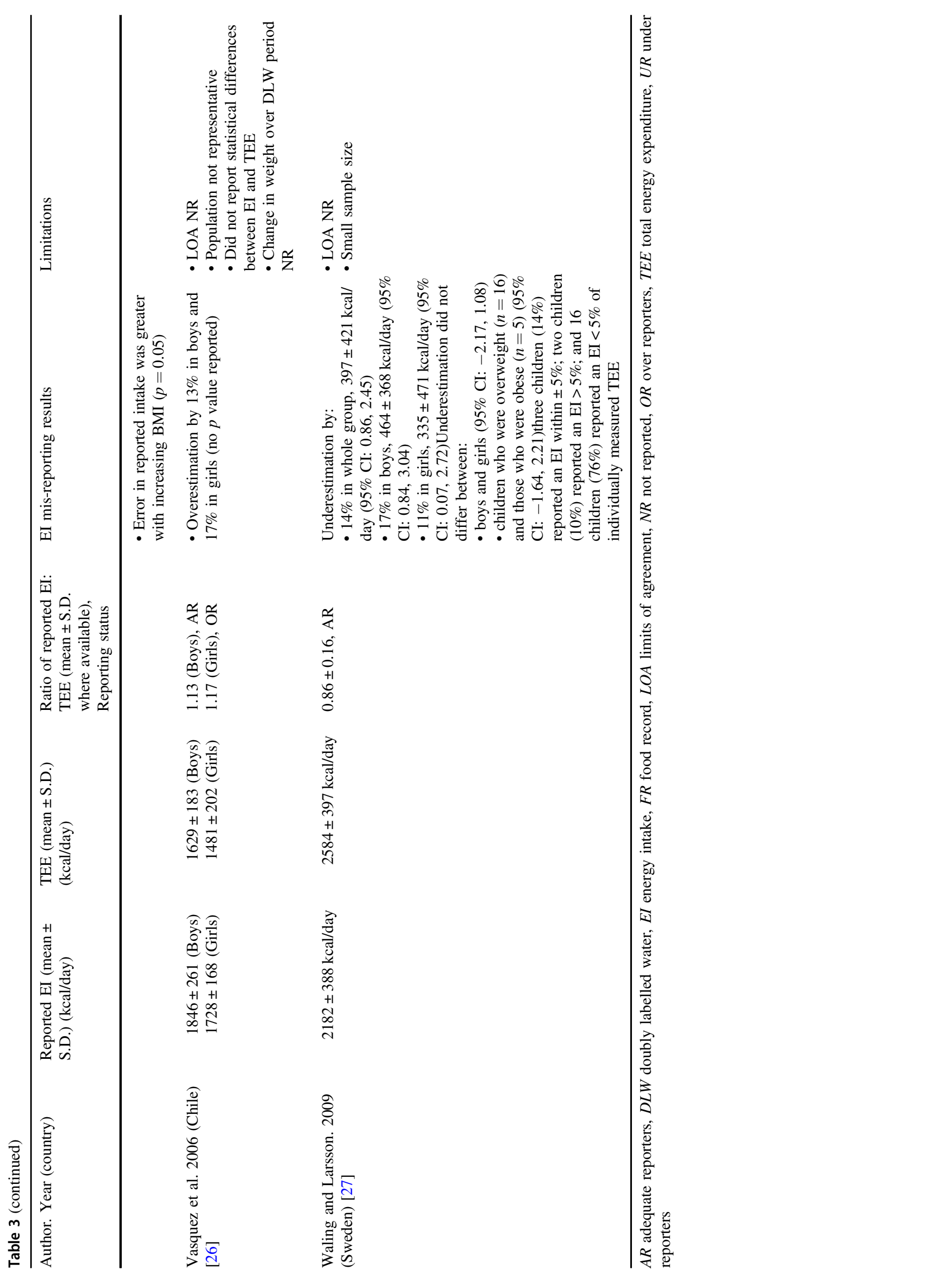




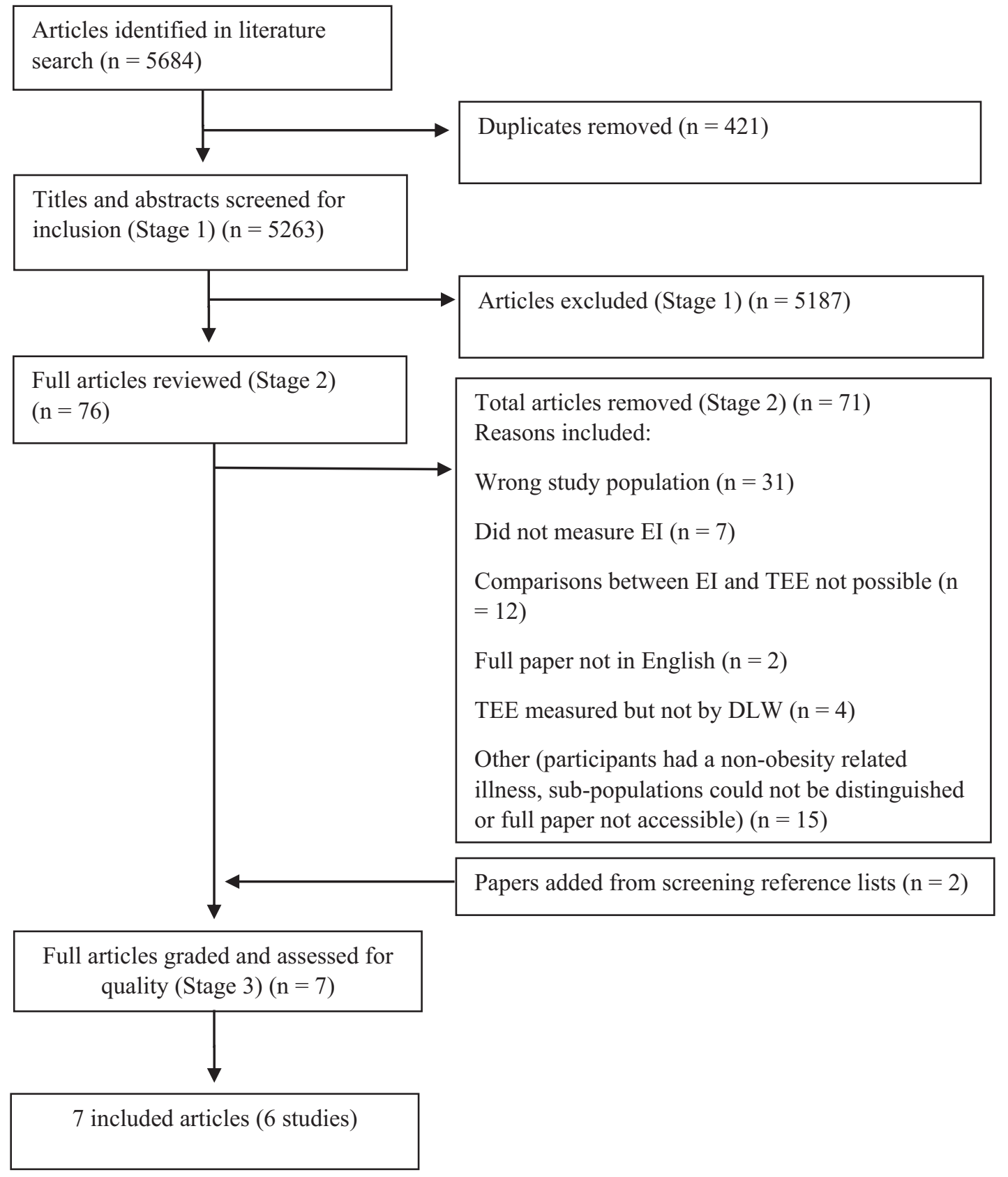

Fig. 1 Flow diagram of searching and inclusion processes

period [23,24]. Table 1 gives an overview of the participant information, weight status classification and DLW details for each article.

All included studies in this review used only one dietary assessment method. Four of the included studies used a food record (FR) for the assessment of EI, ranging in length from 3 to 14 days and all were paper-based. Of these studies, two were used in adolescent populations (12 years and older) and the adolescents were the reporters [21,25], parents and trained nutritionists reported intake in the one study for young children (3-5 years) using a 3-day FR [26], and a combination of parents and children reported intake in an 8day FR where children (9-12 years) were the participants [24]. One study used a diet history interview (DHI) with a trained nutritionist to estimate EI in children (8.3-12.4 years) over the previous 2 weeks, using a combination of parents and children as reporters [27], and the remaining study used two 24-h dietary recalls (24h DR) in a group of children (4-10 years), where parents only were the reporters [23]. A range of techniques were used to account for potential error and increase the accuracy of the data collected from the dietary assessment methods. Training of 
participants in the use of the dietary assessment method $[21,24,25]$, the use of standardised paperwork [21,25], the use of food models/photographs/guides and measuring cups and spoons [21,23-25,27], incorporation of trained professionals in the data collection and analysis phases [21,23-27], increased participant contact during the data collection period $[21,26]$ and the use of appropriate analysis tools (country-specific food composition data and/or suitable software) was common across studies [21,23-27]. Details regarding each dietary assessment method and the particular procedures can be found in Table 2 .

Table 3 describes the results, outcomes and limitations of included studies. When compared to TEE as the validation measure, mis-reporting of EI ranged from -44.5 to $17 \%$ across all studies. EI was found to be both under-reported and over-reported by FR [21,24-26], with the number of recording days having no impact on results. When considering the pre-defined reporting status criteria, overweight and obese participants in all studies using FR were classified as under-reporters. Results from one study by Waling and Larsson [27] detailed that under-reporting by an average of $14 \%$ in their study sample when using a DHI, which was classified as adequate-reporting from the defined criteria. Only one study found over-reporting of EI. Vasquez et al. used a 3-day FR to estimate EI in 3-5 year olds using a combination of parent and trained nutritionist reporting [26]. Results from their cohort showed overestimation by $13 \%$ in boys (classified as adequate reporters) and $17 \%$ in girls (classified as over-reporters); however, the significance of these results was not reported [26].

Under-reporting was evident in the 2014 study by Börnhorst et al, where two $24 \mathrm{~h}$ DR were used to estimate EI [23]. Results, however, are the most accurate of all studies, and detail that EI was underestimated by only $5.5 \%$ in the overweight or obese children, compared to $0.1 \%$ in the thin/normal children [23]. When considering the predefined reporting status criteria, these children were classified as adequate reporters. This study also reported good agreement at the group level for estimates of EI [23].

LOAs were not reported in any studies; therefore, the accuracy of reporting on an individual level could not be determined. Reporting status was found to be influenced by weight status in two studies, both of which included only adolescents who were the reporters [21,25]. Underestimation was greater in the obese group when compared to the non-obese group $(p<0.001)$ when considering one study that used a 14-day FR for estimation of EI [21], and the error in reported intake was greater with increasing body mass index $(p=0.05)$ when using a 9-day FR [25]. Conflicting results were found in the study by Waling and Larsson, where underestimation did not differ between children who were overweight or obese $(95 \%$ confidence interval (CI): $-1.64,2.21)$ when using a DHI and combination of parent and child reporting [27]. The influence of gender on reporting was investigated in two studies, which found significant under-reporting regardless of gender when using either a 9-day FR $(p<0.0001)$ [25] or DHI (95\% CI: $-2.17,1.08)$ [27] to estimate EI (Table 3).

\section{Discussion}

This systematic literature review critiqued six studies to determine the validity of dietary assessment methods when estimating EI in children and adolescents who are overweight or obese. Despite evidence of mis-reporting across all dietary assessment methods, the 24-h DR and DHI were more accurate than others used in this population, detailing adequatereporting and good agreement between estimated EI and measured TEE at the group level. A $24 \mathrm{~h}$ DR was used on two occasions in a group of overweight/obese children and found a high level of agreement between EI and TEE (0.96). The thin/ normal children included in this study also showed a similar level of agreement (1.01) [23]. This reported accuracy is similar to previous literature where $24 \mathrm{~h}$ DR were used to estimate EI in children of varying body weights and detailed mis-reporting from -14 to $11 \%$ [15]. A DHI interview was used to estimate EI in a group of 21 overweight and obese children, and detailed adequate-reporting at the group level and underestimation by $14 \%$ [27].

Of all dietary assessment methods, FRs were the least accurate as evidenced by the lower EI to TEE ratio. Interestingly, the number of recording days for FR did not seem to impact on the accuracy of results. One study that used a 14-day FR detailed poor levels of reporting-underestimation by $41.3 \pm 23.6 \%$ overall [21], and by $44.5 \pm$ $21.8 \%$ in a subset of participants [22]. However, given that all participants were paid to encourage adherence, one would reasonably expect that reporting would be more accurate [21]. Results may reflect participant characteristics (reported bias from adolescents and the idea of socially desirable reporting), or the use of an extended dietary assessment period (in which fluctuations in EI may have been an issue) and associated respondent burden. Use of a 9-day FR and an 8-day FR still resulted in under-reporting, but to a lesser extent than the 14-day FRs $(35 \pm 20$ and 31.5 \pm 2.9 or $24.5 \pm 3.2 \%$ (depending on weight group), respectively) $[24,25]$. The participants in these studies were children and adolescents. All studies using FR employed various measures to increase the accuracy of reporting. Each one used appropriate dietary analysis software or food composition tables, incorporated food models/illustrations/ measuring cups and spoons as guides for determining portion size, trained or instructed participants on how to use a FR and had qualified nutritionists overseeing the procedures and checking in with participants [21,24-26]. Studies using 
the $24 \mathrm{~h}$ DR and DHI also employed similar measures $[23,27]$.

Only one study in this review observed overestimation of EI using a 3-day FR [26]. Trained professionals measured, weighed and checked food intake on day-care centre days, and parents completed the FRs in the evening and on one weekend day. This resulted in overestimations of EI by 13-17\% [26]. Recommendations from the literature suggest that weighed FRs provide the best estimates of EI for younger children aged 0.5-4 years (based on results from children of normal weight) [15]; however, this was not observed in the group of young children who were overweight or obese. It is not clear why the overestimation would occur; however, it is thought that the methodological processes including the fact that there were two distinct but separate reporters could explain this.

The reporter of EI may provide an explanation for the mis-reporting. As mentioned previously, adolescents underestimated their EI when using FR [21,25], which reflects previous literature [28]. In the one study where parents were proxy reporters for their child (4-10 years of age), the reported EI was statistically similar to TEE $(-5.5 \%)$ when measured by $24 \mathrm{~h}$ DR [23]. FR underestimated EI $(31.5 \pm 2.9$ and $24.5 \pm 3.2 \%)$ when the parent and child were combined reporters [24], whereas the DHI had adequate agreement when the parent and child were combined reporters $(-14 \%)$ [27]. Vasquez et al. used a modified 3-day FR where both parents and trained nutritionists were reporters of EI for young children (3-5 years), and was the only study where over-reporting was evident ( $+13-17 \%$ ) [26]. These findings suggest an underreporting nature of adolescents who are overweight or obese, which could be due to a number of factors including engaging in an energy-restrictive diet, variations in EI (whether deliberate or not), socially desirable reporting or selective under-reporting. For example, in the study by Bandini et al. published in 1990, all participants were recruited from tertiary weight control clinics, and it was emphasised to participants that the measurement time frame was a weight maintenance period, and the participants were paid for their time [21]. This bias, or the Hawthorne effect, is evident in research that has assessed children and adolescents [28].

In the three studies that reported results for overweight/ obese participants and non-overweight/non-obese participants, the latter group reported greater agreement of EI with TEE, which was significant in one study [21], and not tested in the remainder $[23,24]$. The dietary assessment methods used in these studies were FR (14 and 8 days) $[21,24]$ and two $24 \mathrm{~h}$ DR [23]. It appears that this inability to accurately report EI in heavier subjects may reflect the mis-reporting nature of this population, rather than the errors in the application of the dietary assessment methods. Although the overweight or obese groups were less accurate reporters of EI compared to normal weight groups, in two of the four studies, all participants were classified into the same category as under-reporters or average reporters [21,23].

Stating the ratio of EI to TEE or mis-reporting percentage highlights agreement at the group level. Nonetheless, only the mean of the whole group is of interest and individual differences are ignored. The LOA was not reported in any studies, which limits the ability to completely comprehend individual differences. It was concluded that a FR was not a reliable method for measuring EI in overweight and obese children and adolescents at the group level [21,24-26]. Alternatively, at this stage, the $24 \mathrm{~h}$ DR and DHI appear to be valid methods at the group level [23,27]; however, further research is required to confirm this.

Previous researchers have suggested that there is a need to develop new dietary assessment methods that incorporate specific measures to help overcome the respondent reporting error, such as better training of subjects, the use of technology and methods that require less burden [25,29]. With the increasing use of personal mobile technologies, such advancements are occurring (such as mobile FRs and the use of wearable cameras), with relative validity being established [30,31], but comparisons to measured TEE via DLW are yet to be done. While these upcoming advancements in dietary assessment are promising, it is highly likely that the inherent nature of people to under-report will never be overcome by any dietary assessment methods where participants are aware that they are being monitored. Researchers need to be aware of the day-to-day variation in EI in the population of interest, establish clinically meaningful points and understand the degree of under-reporting present in that population, even when using tools that incorporate objective aspects. Under-reporting in adolescents remains a challenge, with a need for more qualitative research investigating and understanding the psychosocial aspects of dietary intake.

One major limitation is the lack of consistency when classifying overweight or obese. Five different classification methods were used across the six studies, increasing the uncertainty when comparing study populations. Ideally, future research would focus on a consistent method for classifying overweight and obesity in order for results to be comparable. Only articles that were published in English were included, which is another limitation. While preliminary conclusions can be drawn, these are based on limited statistical data from a small collection of studies, all of which lack power calculations. The majority of studies $(n=4)$ included a small sample number $(n<30)$ of overweight or obese participants (view Table 1), and only few studies used each dietary assessment method. This is understandable, given the high costs and participant burden of these types of validation studies, but identifies a clear 
need for ongoing research. Errors and bias associated with the included dietary assessment methods also impact on the findings of this review. In addition, all studies in this review were level III-2 evidence [18], primarily due to the fact that blinded comparisons did not occur, and the use of convenience samples, but this is understandable, given the constraints and processes required in the DLW technique.

After extensively reviewing the literature, it is evident that dietary assessment method validation studies are lacking, especially those that include children and adolescents who are overweight or obese. Thus, the need to modify available dietary assessment methods, develop new dietary assessment methods that can overcome the mis-reporting nature of overweight and obese children and adolescents and validate these methods to provide accurate agreement at the individual level is increasingly important.

This review aimed to determine the validity of dietary assessment methods to accurately measure EI in children and adolescents who are overweight or obese. A total of six studies from seven articles were identified and critically analysed. On the basis of the few studies identified in this review, the evidence is not conclusive as to the most accurate method to use in children and adolescents who are overweight or obese. From the six studies, the $24 \mathrm{~h}$ DR and DHI are reported to be the most accurate dietary assessment methods for children aged 4-14 years where either parents or a combination of children and parents are the reporters. The results from this review should be considered carefully in the context given overall the lack of studies in this area using the DLW method. Overall, good agreement was found at the group level; however, researchers and clinicians need to be aware of individual variation. Furthermore, FRs were repeatedly inaccurate across all age groups and reporters, and at this stage should not be used for the assessment of EI in children and adolescents who are overweight or obese. It is evident that adolescents who are overweight or obese under-report to a greater extent when compared to normal weight adolescents; however, it is not yet clear which dietary assessment method is best to use for adolescents or even young children who are overweight or obese. Further research is necessary to aid in the development and validation of accurate dietary assessment methods (particularly when considering emerging technologies), or ways to improve existing measures when assessing EI in children and adolescents who are overweight or obese.

Conflict of interest The authors declare that they have no competing interests.

Open Access This article is licensed under a Creative Commons Attribution-NonCommercial-NoDerivatives 4.0 International License, which permits any non-commercial use, sharing, distribution and reproduction in any medium or format, as long as you give appropriate credit to the original author(s) and the source, and provide a link to the
Creative Commons license. You do not have permission under this license to share adapted material derived from this article or parts of it. The images or other third party material in this article are included in the article's Creative Commons license, unless indicated otherwise in a credit line to the material. If material is not included in the article's Creative Commons license and your intended use is not permitted by statutory regulation or exceeds the permitted use, you will need to obtain permission directly from the copyright holder. To view a copy of this license, visit http://creativecommons.org/licenses/by-nc-nd/4.0/.

\section{References}

1. World Health Organisation. Childhood overweight and obesity. 2017. http://www.who.int/dietphysicalactivity/childhood/en/. Accessed 9 Jan 2017.

2. Australian Bureau of Statistics. National health survey first results Australia 2014-2015. Canberra; 2015. p. 56. ABS Cat. No. 4364.0.55.001.

3. National Health and Medical Research Council. Clinical practice guidelines for the management of overweight and obesity in adults, adolescents and children in Australia. Melbourne: National Health and Medical Research Council; 2013. p. 232.

4. Wang Z, Patterson CM, Hills AP. The relationship between BMI and intake of energy and fat in Australian youth: a secondary analysis of the National Nutrition Survey 1995. J Nutr Diet. 2003;60:23-29.

5. Hassapidou M, Fotiadou E, Maglara E, Papadopoulou SK. Energy intake, diet composition, energy expenditure, and body fatness of adolescents in northern Greece. Obesity. 2006;14:855-62.

6. Labonté M, Kirkpatrick SI, Bell RC, Boucher BA, Csizmadi I, Koushik A, et al. Dietary assessment is a critical element in health research - perspective from the partnership for advancing nutritional and dietary assessment in Canada. Appl Physiol Nutr Metab. 2016;41:1096-9.

7. Subar AF, Freedman LS, Tooze JA, Kirkpatrick SI, Boushey C, Neuhouser ML, et al. Addressing current criticism regarding the value of self-report dietary data. $J$ Nutr. 2015;145:2639-45. https://doi.org/10.3945/jn.115.219634

8. Thompson FE, Subar AF, Loria CM, Reedy JL, Baranowski T. Need for technological innovation in dietary assessment. $J \mathrm{Am}$ Diet Assoc. 2010;110:48-51.

9. Magarey A, Watson J, Golley RK, Burrows T, Sutherland R, McNaughton SA, et al. Assessing dietary intake in children and adolescents: Considerations and recommendations for obesity research. Int J Pediatr Obes. 2011;6:2-11.

10. Block G, Hartman AM. Issues in reproducibility and validity of dietary studies. Am J Clin Nutr. 1989;50:1133-8.

11. Gibson RS. Principles of nutritional assessment. 2nd edn. Oxford University Press: NY, USA, 2005. p. 908.

12. Speakman JR. The history and theory of the doubly labeled water technique. Am J Clin Nutr. 1998;68:932S-38S.

13. Schoeller DA, Fjeld CR. Human energy metabolism: what have we learned from the doubly labeled water method? Annu Rev Nutr. 1991;11:355-73.

14. Kuzawa CW. Adipose tissue in human infancy and childhood: an evolutionary perspective. Yearb Phys Anthropol. 1998;41:177-209.

15. Burrows TL, Martin RJ, Collins CE. A systematic review of the validity of dietary assessment methods in children when compared with the method of doubly labeled water. J Am Diet Assoc. 2010;110:1501-1510.

16. Coulston AM, Boushey CJ, Ferruzzi M. Nutrition in the prevention and treatment of disease. Elsevier/Academic: USA, 2013. 
17. Liberati A, Altman DG, Tetzlaff J, Mulrow C, Gotzsche PC, Ioannnidis JPA, et al. The PRISMA statement for reporting systematic reviews and meta-analyses of studies that evaluate health care interventions: explanation and elaboration. PLoS Med. 2009; 6:e1000100.

18. National Health and Medical Research Council. NHMRC additional levels of evidence and grades for recommendations for developers of guidelines. Australia, 2009. p. 23.

19. Academy of Nutrition and Dietetics. Evidence analysis manual: steps in the academy evidence analysis process; Chicago, USA, 2012. p. 111.

20. Sjoberg A, Slinde F, Arvidsson D, Ellegard L, Gramatkovski E, Hallberg L, et al. Energy intake in Swedish adolescents: validation of diet history with doubly labelled water. Eur J Clin Nutr. 2003;57:1643-52.

21. Bandini LG, Schoeller DA, Cyr HN, Dietz WH. Validity of reported energy intake in obese and nonobese adolescents. Am J Clin Nutr. 1990;52:421-5.

22. Bandini LG, Vu D, Must A, Cyr H, Goldberg A, Dietz WH. Comparison of high-calorie, low-nutrient-dense food consumption among obese and non-obese adolescents. Obes Res. 1999; 7:438-43.

23. Börnhorst C, Bel-Serrat S, Pigeot I, Huybrechts I, Ottavaere C, Sioen I, et al. Validity of 24-h recalls in (pre-) school aged children: Comparison of proxy-reported energy intakes with measured energy expenditure. Clin Nutr. 2014;33:79-84.

24. Champagne CM, Baker NB, DeLany JP, Harsha DW, Bray GA. Assessment of energy intake underreporting by doubly labeled water and observations on reported nutrient intakes in children. J Am Diet Assoc. 1998;98:426-33.

25. Singh R, Martin BR, Hickey Y, Teegarden D, Campbell WW, Craig BA, et al. Comparison of self-reported and measured metabolizable energy intake with total energy expenditure in overweight teens. Am J Clin Nutr. 2009;89:1744-50.

26. Vásquez F, Salazar G, Andrade M, Vásquez L, Diaz E. Energy balance and physical activity in obese children attending day-care centres. Eur J Clin Nutr. 2006;60:1115-21.

27. Waling MU, Larsson CL. Energy intake of Swedish overweight and obese children is underestimated using a diet history interview. J Nutr. 2009;139:522-7.

28. Livingstone MB, Robson PJ, Wallace JM. Issues in dietary intake assessment of children and adolescents. Br J Nutr. 2004;92: S213-222.

29. Rollo ME, Williams RL, Burrows T, Kirkpatrick SI, Bucher T, Collins CE. What are they really eating? A review on new approaches to dietary intake assessment and validation. Curr Nutr Rep. 2016;5:307-314.

30. Burrows TL, Rollo ME, Williams R, Wood LG, Garg ML, Jensen $\mathrm{M}$ et al. A systematic review of technology-based dietary intake assessment validation studies that include carotenoid biomarkers. Nutrients. 2017;9. https://doi.org/10.3390/nu9020140.

31. Gemming L, Utter J, Ni Mhurchu C. Image-assisted dietary assessment: a systematic review of the evidence. J Acad Nutr Diet. 2015;115:64-77.

32. Cole TJ, Bellizzi MC, Flegal KM, Dietz WH. Establishing a standard definition for child overweight and obesity worldwide: international survey. Br Med J. 2000;320:1240-3.

33. Centers for Disease Control and Prevention. U.S. Department of Health and Human Services. https://www.cdc.gov/growthcharts/. Updated 9 Sept 2010; cited 14 Feb 2017.

34. WHO (World Health Organization Working Group). Use and interpretation of anthropometric indicators of nutritional status. Bull World Health Organ. 1986;64:929-41. 